\title{
QUEEN'S
UNIVERSITY
BELFAST
}

\section{An Experimental Investigation into the Impact of Vehicular Traffic on Interpersonal Wearable-to-Wearable Communications Channels}

Doone, M. G., Cotton, S. L., \& Oestges, C. (2017). An Experimental Investigation into the Impact of Vehicular Traffic on Interpersonal Wearable-to-Wearable Communications Channels. IEEE Transactions on Antennas and Propagation, 65(10), 5418-5430. [8039240]. https://doi.org/10.1109/TAP.2017.2738071

Published in:

IEEE Transactions on Antennas and Propagation

Document Version:

Publisher's PDF, also known as Version of record

Queen's University Belfast - Research Portal:

Link to publication record in Queen's University Belfast Research Portal

Publisher rights

Copyright 20_17the authors.

This is an open access article published under a Creative Commons Attribution License (https://creativecommons.org/licenses/by/4.0/), which permits unrestricted use, distribution and reproduction in any medium, provided the author and source are cited.

\section{General rights}

Copyright for the publications made accessible via the Queen's University Belfast Research Portal is retained by the author(s) and / or other copyright owners and it is a condition of accessing these publications that users recognise and abide by the legal requirements associated with these rights.

Take down policy

The Research Portal is Queen's institutional repository that provides access to Queen's research output. Every effort has been made to ensure that content in the Research Portal does not infringe any person's rights, or applicable UK laws. If you discover content in the Research Portal that you believe breaches copyright or violates any law, please contact openaccess@qub.ac.uk. 


\title{
An Experimental Investigation into the Impact of Vehicular Traffic on Interpersonal Wearable-to-Wearable Communications Channels
}

\author{
Michael G. Doone, Student Member, IEEE, Simon L. Cotton, Senior Member, IEEE, \\ and Claude Oestges, Senior Member, IEEE
}

\begin{abstract}
In this paper, we have investigated the effects of vehicular traffic on interpersonal wearable-to-wearable (W2W) communications channels in an urban environment at $2.45 \mathrm{GHz}$. In particular, we have studied the perturbations in the received signal caused by different types of vehicles as they passed through a channel between two persons who maintained various relative orientations while positioned at the opposite sides of a road. As the channel underwent different fading mechanisms depending on whether the vehicle was approaching, transitioning (i.e., intersecting the direct signal path), or receding from the persons, the overall disturbance was appropriately segmented depending on the journey stage. The results have shown that relative body orientation was a significant factor when considering the impact that a vehicle can have on a W2W link. When both persons faced the oncoming traffic, the link was particularly susceptible to significant fading events with variations in the received signal power from the unperturbed state as great as $44.1 \mathrm{~dB}$ observed to occur. For all of the journey stages, irrespective of the relative orientation of the persons, the logarithmically transformed longterm fading process was found to be multimodal and well described by a Gaussian mixture model. During the transitioning phase, shadowing caused by the passing automobile obstructing the line-of-sight signal path was found to be the main contributor to the signal fading. However, probably the most remarkable result of the channel characterization work conducted in this paper was the severity of the short-term fading often observed. Such was the intensity of the measured envelope fluctuation in many of the scenarios, we have been able to utilize the recently proposed $\kappa-\mu$ extreme distribution with great success and in the process, provide a further important empirical validation of this new fading model. Moreover, we have used the resistor-average distance, which is derived from the Kullback-Leibler distance to show the improved fit that the $\kappa-\mu$ extreme distribution offers compared with the $\kappa-\mu$ distribution when used to model the W2W channel in this fading environment.
\end{abstract}

Manuscript received April 14, 2016; revised March 10, 2017; accepted July 26, 2017. Date of publication September 18, 2017; date of current version October 5, 2017. This work was supported in part by the Engineering and Physical Sciences Research Council under Grant EP/L026074/1 and in part by the Interuniversity Attraction Poles Programme 7/23 BESTCOM initiated by the Belgian Science Policy Office. (Corresponding author: Simon L. Cotton.)

M. G. Doone and S. L. Cotton are with the Wireless Communications Laboratory, Institute of Electronics, Communications and Information Technology, Queen's University Belfast, Belfast BT3 9DT, U.K. (e-mail: mdoone03@qub.ac.uk; simon.cotton@qub.ac.uk).

C. Oestges is with the Institute of Information and Communication Technologies, Electronics, and Applied Mathematics, Université Catholique de Louvain, 1348 Louvain-la-Neuve, Belgium (e-mail: claude.oestges@uclouvain.be).

Color versions of one or more of the figures in this paper are available online at http://ieeexplore.ieee.org.

Digital Object Identifier 10.1109/TAP.2017.2738071
Index Terms- $\kappa-\mu$ distribution, $\kappa-\mu$ extreme distribution, channel measurements, channel modeling, fading channels, Gaussian mixture model (GMM), wearable communications.

\section{INTRODUCTION}

$\mathbf{R}$ ECENT years have seen the embracement of smart devices designed to be carried or worn by people. These devices, which are now commonly referred to as "wearables" have the potential to transform how society interacts. With widespread adoption, intercommunication between wearable devices will lead to the creation of vast people-driven networks facilitated by wearable-to-wearable (W2W) communications [1]. These networks will be expected to facilitate not only the sharing of information between users but also the relayed transmission of data meant for other people and networks [2]-[4]. The design of wearable devices and the implementation of robust networking are particularly challenging for wireless systems designed to operate in close proximity to the human body [5]. This occurs for two main reasons: first, the electromagnetic interaction effects between human tissue and RF circuitry, including the antenna [6], and also due to the unique geometry of the propagation problem. Wireless systems involving only humans are typically used at low elevations and are, therefore, susceptible to shadowing and fading events caused not only by the wearer's body but also other pedestrians and obstacles in the local vicinity [7].

For example, in so-called "off-body" communications, the shadowing caused by the body obstructing the signal is often responsible for significant fading and reduction in channel reliability [8]-[10]. At microwave frequencies, the movement of a limb across the line-of-sight (LOS) signal path can induce channel fades as great as $20 \mathrm{~dB}$ [10]. The disruption to signal propagation caused by nearby moving objects was investigated in [11] and [12]. In [11], experiments were conducted, which considered the "on-body" channels found in body area networks in the presence of a walking passerby. The results concluded that the presence of another person, despite not directly obstructing the main signal propagation path, still had a significant impact, with the fades of greater than $20 \mathrm{~dB}$ observed. Similarly, the human-induced shadowing effect for a person walking through a link was investigated in [12]. The duration of a single fading event attributable to body shadowing was observed to last around $550 \mathrm{~ms}$ with the mean 
attenuation between 6 and $18 \mathrm{~dB}$. From these studies, it is clear that objects moving in the vicinity of wearable communication systems have a substantial impact on the channel performance. Therefore, from a system design perspective, it is essential that the magnitude of the potential channel impairments caused by objects surrounding the wearable device user is adequately characterized and understood.

Moving beyond the above-discussed studies, consumers will expect wearable devices to operate not only indoors, but also in a wide variety of outdoor environments, such as city centers and urban areas. In these locations, moving objects will not just be limited to pedestrians, but will also include vehicles which may be operating in close proximity. In vehicle-tovehicle (V2V) communications, the relatively low heights of the antennas on vehicles can result in possible obstruction of the LOS signal by either stationary objects (e.g., buildings, trees, and road signs) or mobile objects (e.g., other moving vehicles). This has led to deterministic- and stochastic-based studies to investigate the effect of shadowing within vehicular networks [13]-[19].

In [14] and [15], experiments were conducted to quantify the impact of obstructing vehicles that block the LOS between two cars communicating as part of a vehicular network. The results concluded that at $10 \mathrm{~m}$, a van obstructing the channel can cause a $20 \mathrm{~dB}$ attenuation in the received signal power. This was observed within both the 2.45 and $5.8 \mathrm{GHz}$ frequency ranges. The severity of the signal attenuation was observed to reduce as the separation distance between vehicles was increased. At twice the separation distance $(20 \mathrm{~m})$, the received signal power was observed to decrease by $12 \mathrm{~dB}$. At the same distance, the author reported a larger truck caused a significant $27 \mathrm{~dB}$ decrease in the received signal power. In [15], the theory of multiple knife-edge diffraction was used to model the shadowing effect of the vehicles. However, the results concluded that the model only provided adequate estimates of the signal attenuations for the larger separation distances. An extensive measurement campaign was performed by He et al. [16] on the shadowing effects caused by a bus on a similar vehicular channel. They reported signal attenuations between 15 and $20 \mathrm{~dB}$, which are comparable with the results in [14] and [15].

The measurements performed in [14]-[16] only considered the shadowing effects caused by a vehicle that obstructed the channel between two vehicles forming a V2V communications link. However, in [13], the channel between a stationary car and a base station situated by the roadside was investigated, as a large truck passed across the direct signal path between the communicating nodes. Similar to [15], the theory of knife-edge diffraction was used to estimate the signal attenuation across a range of frequencies (from $1 \mathrm{GHz}$ to $40 \mathrm{GHz}$ ). The authors simplified the model of the truck by considering the effect of the cab to be negligible and ignoring the signal propagation underneath the trailer. Measurements were performed to validate the model at $11 \mathrm{GHz}$, and signal attenuations were found to be as significant as $35 \mathrm{~dB}$ compared with the unobstructed link.

Previous work in [20] and [21] has presented some initial results pertaining to the influence of vehicular traffic on the
W2W channel. In [20], the impact of uncontrolled traffic on a W2W link between two persons situated on the opposite sides of a road was investigated, however, this was restricted to short-term fading only. Later in [21], controlled traffic flow was considered, and the analysis of the data extended to report the maximum fade depth and the duration of channel disturbance, though this paper did not present any statistical modeling. To the best of our knowledge, a systematic study of vehicle-induced shadowing and fading in human centric channels has yet to be reported in the open literature. In the sequel, we significantly extend the work conducted in [20] and [21] by providing an in-depth statistical characterization of the fading caused by realistic traffic flow occurring in the vicinity of a W2W link. Most notably, we perform a categorization of the passing vehicles based on their size enabling us to quantitatively report on the channel disturbances induced by the traffic. More precisely, we report the maximum fade induced and the length of time that the channel is influenced by differing vehicular types. Furthermore, we consider various relative body orientations of the two persons forming the W2W link to determine the role of human body shadowing in W2W communications, which are subject to vehicular traffic. Due to the nonstationary behavior of the W2W channel, we segment our data into approaching, transitioning, and receding phases. We characterize the long-term fading using a Gaussian mixture model (GMM) and the short-term fading using the $\kappa-\mu$ and later the $\kappa-\mu$ extreme distributions.

The remainder of this paper is organized as follows. Section II outlines the measurement system, the experimental environment, and the various scenarios investigated in this paper. Section III presents the methodology for the classification of the vehicle types considered in this paper. In Section IV, some metrics utilized for quantifying the impact of vehicular traffic on the $\mathrm{W} 2 \mathrm{~W}$ channel are introduced and used to compare the influence of different vehicle types and body orientations. Sections V and VI present a statistical characterization of the long-term and the short-term fading, respectively. Finally, Section VII completes this paper with some concluding remarks.

\section{Measurement System, EnVironment, AND PROCEDURE}

\section{A. Measurement System}

The measurement system used in this paper consisted of a number of purposely developed $2.45 \mathrm{GHz}$ wireless sensor nodes. The transceiver section of each node featured an ML2730, a single chip fully integrated frequency shift keyed transceiver, manufactured by RF Micro Devices. When operating as a transmitter, the node was configured to transmit a continuous wave signal with an output power of $+17.6 \mathrm{dBm}$. The transceivers were controlled using dedicated PIC32MX microcontrollers, manufactured by Microchip Technology Inc. When operating as a receiver, the microcontroller measured the receive signal strength (RSS) at a rate of $10 \mathrm{kHz}$ using the onboard 10-bit analog-to-digital (ADC) converter. To improve the sampling signal-to-noise ratio, ten ADC measurements were obtained for each RSS sample, and then averaged before 


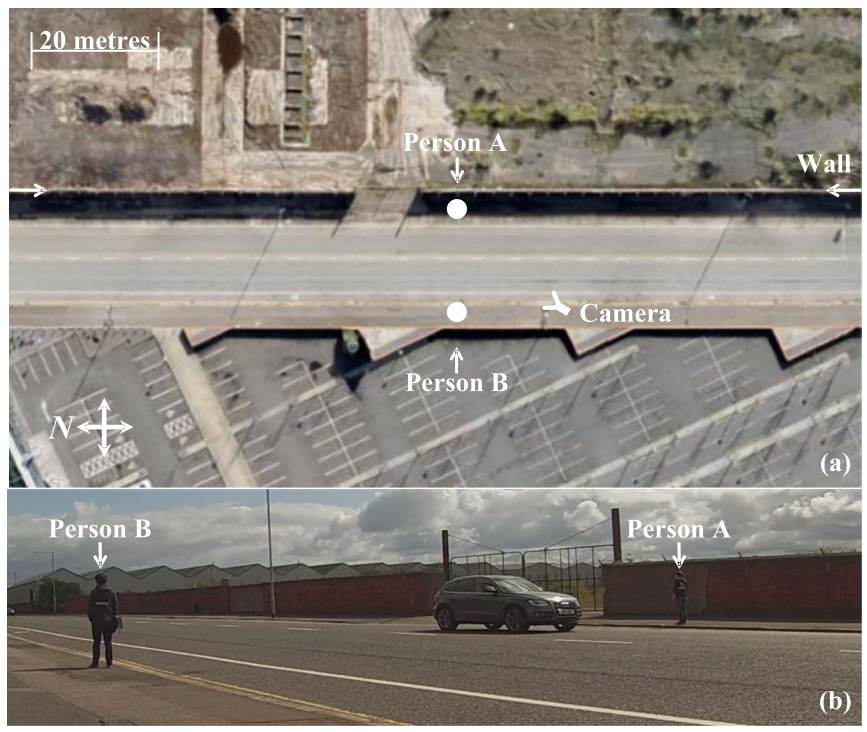

Fig. 1. (a) Satellite view of the measurement environment highlighting the positions of both persons and an HD video camera that was used to record the passing traffic (image courtesy of Google Maps), (b) Snapshot from the camera when a vehicle was passing.

logging. The receiving node was precalibrated using a Rohde \& Schwarz SMU200A vector signal generator. Approximately one million RSS samples were collected during the measurement campaigns presented in this paper. It should be noted that prior to beginning the measurement scenarios, the average noise threshold was determined and found to be $-91.7 \mathrm{dBm}$. The antennas used for this paper were $+2.3 \mathrm{dBi}$ gain omnidirectional sleeve dipole antennas (Mobile Mark model PSKN3-24/55S). They were connected directly to the front end of the transceivers using a SubMiniature version A connector and maintained a vertically polarized orientation throughout.

\section{B. Measurement Environment and Procedure}

To facilitate a study of the impact that different vehicles can have on the W2W channel, the measurement location chosen for this paper was a road situated within a busy business district in the Titanic Quarter of Belfast, U.K., as shown in Fig. 1(a). The measurement environment consisted of a straight stretch of road, with a wall approximately $2 \mathrm{~m}$ in height that ran along the sidewalk near person A [visible in Fig. 1(b)]. The road had a maximum speed limit of $30 \mathrm{~m} / \mathrm{h}$ (or equivalently $13.4 \mathrm{~ms}^{-1}$ ), and the traffic flow was uncontrolled for the duration of the measurements. The transmitter and the receiver were placed on the central chest region of two persons $\mathrm{A}$ and $\mathrm{B}$, respectively, using a $5 \mathrm{~mm}$ dielectric spacer consisting of Rohacell HF 51 foam $\left(\epsilon_{r}=1.07\right)$. Person A had a mass of $80 \mathrm{~kg}$ and a height of $1.72 \mathrm{~m}$, and person B had a mass of $75 \mathrm{~kg}$ and a height of $1.83 \mathrm{~m}$. Fig. 2 shows the azimuthal radiation patterns measured in an anechoic chamber for the sleeve dipole antenna in a free space and when situated on persons A and B. Due to the presence of the user's bodies, we can observe a reduction in the antenna's gain in the lateral directions by as much as $20 \mathrm{~dB}$ (i.e., at $90^{\circ}$ and $270^{\circ}$ ).

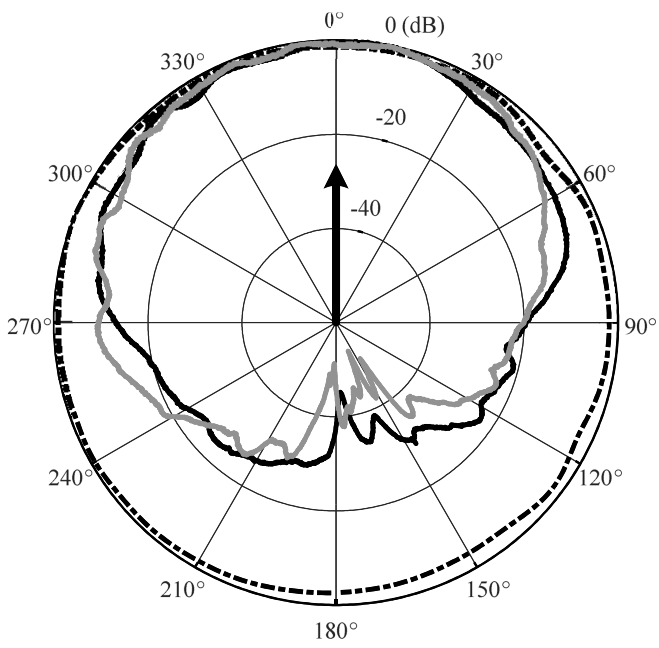

Fig. 2. Measured azimuthal radiation patterns for the sleeve dipole antenna in a free space (black dashed lines) and when situated on person A (gray continuous line) and person B (black continuous line). It should be noted that the black arrow indicates the direction that both persons were facing.

These patterns were further distorted to the posterior of the test subject's bodies, with a decrease in the antenna radiation patterns of up to $53 \mathrm{~dB}$ observed due to body shadowing when compared with the radiation pattern in a free space.

The measurements were conducted for three different relative body orientations, which were deemed typical for wearable systems operating in this environment. Fig. 3 shows these three scenarios with arrows indicating the orientation of the person's bodies while forming the W2W link. It should be noted, to isolate the influence of traffic moving in the vicinity of the W2W channel, the bodies remained stationary in all scenarios. During Scenario A, both persons stood parallel on the opposite sides of the road, facing in the direction of oncoming traffic. In this orientation, both bodies will partially shadow the channel due to the shoulder region obscuring the direct signal path between the hypothetical wearable devices. For comparison, Scenario B involved both persons on the opposite sides of the road while facing one another, thus permitting a LOS signal path between the bodies (unless obstructed by traffic). Last, Scenario C considered the case where both persons were facing in the opposite direction, imitating the situation in which pedestrians travel in the opposite directions on the roadside. During the measurements, a highdefinition (HD) digital video camera was used to record all passing traffic, allowing easy identification of different vehicle types and permitting their passing to be time synchronized to the measurements [the location of the camera is shown in Fig. 1(a)].

\section{Vehicle ClassificAtion}

The internationally recognized vehicle classification system is defined in ISO 3833-1997 (International Organization for Standardization) [22]. In [22], vehicles are grouped based on their structure and design. However, in the USA, another classification scheme is used by the Environmental Protection Agency (EPA) [23]. This scheme uses the vehicle's total 


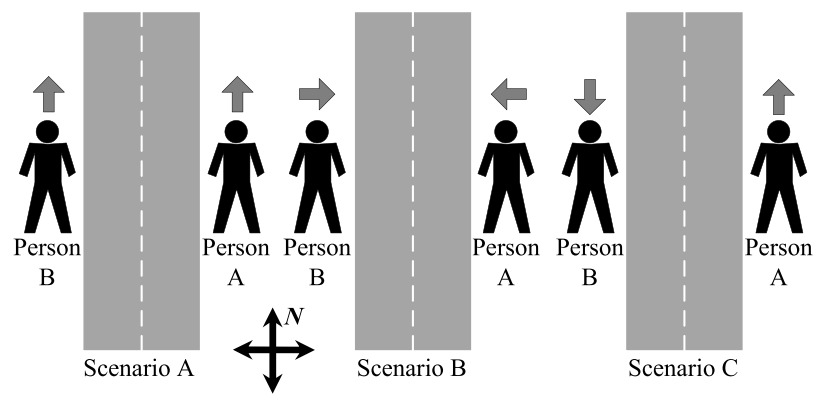

Fig. 3. Measurement scenarios considered in this paper, with arrows highlighting the relative body orientations.

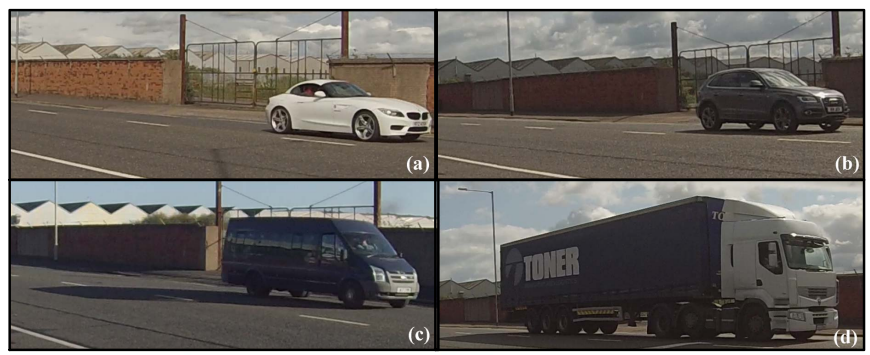

Fig. 4. Example snapshots captured from the HD camera showing (a) Type I, (b) Type II, (c) Type III, and (d) Type IV vehicles.

interior passenger and cargo volumes only to perform the grouping. By comparing across both classification lists, in this paper, we categorize the vehicles, which are typically found in urban environments into one of the four groups. These are as follows.

Type I: It includes any small passenger car but excludes multiperson vehicles (minivans in USA). These vehicles are classified in section 3.1.1 of the ISO standard, and in section 1 of the EPA standard (e.g., Ford Fiesta and Audi A1).

Type II: It includes multiperson vehicles (excluding large pickup trucks) and any other vehicle of the same size (e.g., small vans). These vehicles are classified in section 2 of the U.S. EPA standard (e.g., Opel Zafira and Volkswagen Caddy Van).

Type III: It includes light commercial vehicles, such as large vans and large pickup trucks. These vehicles are also described in section 2 of the U.S. EPA standard (e.g., Ford Transit and Renault Master).

Type IV: It includes buses and lorries (or trucks in USA) and any other vehicles that are larger than Type III. These vehicles are defined in sections 3.1.1 and 3.1.2 of the ISO standard (e.g., Mercedes-Benz Axor and Iveco Eurocargo).

Examples of each type of vehicle obtained from the video footage recorded during the measurements are shown in Fig. 4.

\section{SOME EMPIRICAL OBSERVATIONS}

In this paper, to quantify and compare the effects of vehicleinduced fading and shadowing on the $\mathrm{W} 2 \mathrm{~W}$ channel, we now introduce some metrics that were used to empirically characterize the data. Fig. 5 shows an exemplary received signal power time series that was obtained for a Type I vehicle, as it

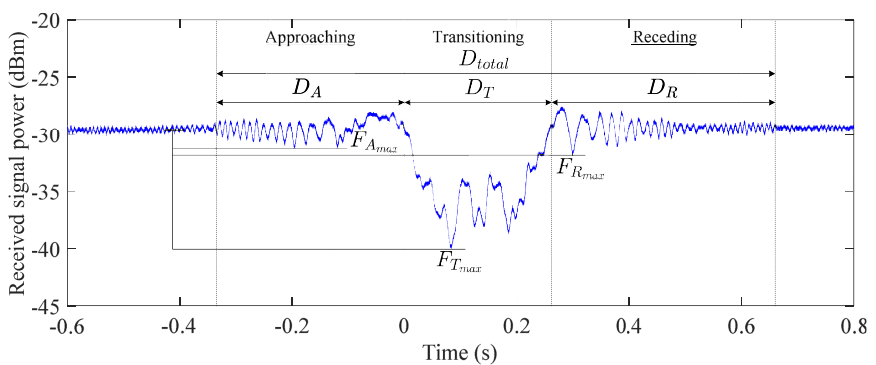

Fig. 5. Received signal power time series for a Type I vehicle passing through the W2W channel during Scenario B.

traveled through the W2W channel during Scenario B. ${ }^{1}$ As we can quite clearly see, the channel exhibited a different fading behavior dependent upon where the vehicle was relative to the W2W link. For example, as the vehicle approached the two persons, noticeable perturbations in the received signal power were observed to occur approximately $334 \mathrm{~ms}$ before the vehicle began to intersect the direct signal path. Herein, this phase of the vehicle's journey is referred to as the approaching region (Fig. 5). The most significant variations in the channel occurred as the vehicle obscured the direct signal path between the two persons, which is denoted as the transitioning region. Upon exiting the transitioning region, there was also an observable disturbance in the channel-this segment of the journey is referred to as the receding region. Beyond these three regions, the channel was considered as being in an unperturbed or "normal" state. It is worth highlighting that these characteristics were readily identifiable across all scenarios for all vehicle types, albeit with different magnitudes. Furthermore, as we shall show in the sequel, not only did the duration of each of these three regions vary dependent upon the relative body orientation, but their channel statistics were also very different, requiring a separate analysis.

To compare the magnitude of the fades and duration of the disturbances induced by different vehicle types over each of the three phases, we defined two quantities, namely, the maximum fade within each region and the duration of disturbance. The maximum fade is defined as the maximum decrease in signal power compared with the mean signal power in the unperturbed state (i.e., no vehicular impact), and the duration of the disturbance is defined as the time span for which the channel experiences similar fading characteristics (i.e., the above-outlined regions). The maximum fade and the duration of the approaching, transitioning, and receding regions are accordingly represented by $\left(F_{A_{\max }}\right.$ and $\left.D_{A}\right),\left(F_{T_{\max }}\right.$ and $\left.D_{T}\right)$, and $\left(F_{R_{\max }}\right.$ and $\left.D_{R}\right)$, respectively, and are visually annotated in Fig. 5 and provided in Table I. For brevity, we only discuss the aforementioned parameters, however, the mean $\left(F_{A_{\mu}}, F_{T_{\mu}}\right.$, and $\left.F_{R_{\mu}}\right)$ and standard deviation $\left(F_{A_{\sigma}}, F_{T_{\sigma}}\right.$, and $F_{R_{\sigma}}$ ) of the fades for each region are also provided in Table I, along with the total duration of channel disturbance $\left(D_{\text {total }}\right)$ from the unperturbed state and the total time ( $\left.T_{\text {total }}\right)$ considered to contain a vehicle passing event.

\footnotetext{
${ }^{1}$ For all received signal power time series, $0 \mathrm{~s}$ is considered as the time at which the vehicle began to intersect the direct path between the two persons.
} 
TABLE I

Duration of Disturbance and Fading Metrics of Each Region for All Scenarios

\begin{tabular}{|c|c|c|c|c|c|c|c|c|c|c|c|c|c|c|c|c|}
\hline \multirow{2}{*}{ Scenario } & \multirow{2}{*}{$\begin{array}{l}\text { Vehicle } \\
\text { Type }\end{array}$} & \multirow{2}{*}{$\begin{array}{l}\text { Unperturbed } \\
\text { Signal } \\
\text { Power } \\
\text { (dBm) }\end{array}$} & \multicolumn{4}{|c|}{ Approaching Region } & \multicolumn{4}{|c|}{ Transitioning Region } & \multicolumn{4}{|c|}{ Receding Region } & \multirow[b]{2}{*}{$\begin{array}{c}D_{\text {total }} \\
(\mathrm{ms})\end{array}$} & \multirow[b]{2}{*}{$\begin{array}{c}T_{\text {total }} \\
(\mathrm{ms})\end{array}$} \\
\hline & & & $\begin{array}{l}D_{A} \\
(\mathrm{~ms})\end{array}$ & $\begin{array}{c}F_{A_{\max }} \\
(\mathrm{dB})\end{array}$ & $\begin{array}{l}F_{A_{\mu}} \\
(\mathrm{dB})\end{array}$ & $\begin{array}{l}F_{A_{\sigma}} \\
(\mathrm{dB})\end{array}$ & $\begin{array}{l}D_{T} \\
(\mathrm{~ms})\end{array}$ & $\begin{array}{c}F_{T_{\max }} \\
(\mathrm{dB})\end{array}$ & $\begin{array}{l}F_{T_{\mu}} \\
(\mathrm{dB})\end{array}$ & $\begin{array}{l}F_{T_{\sigma}} \\
(\mathrm{dB})\end{array}$ & $\begin{array}{c}D_{R} \\
(\mathrm{~ms})\end{array}$ & $\begin{array}{c}F_{R_{\max }} \\
(\mathrm{dB})\end{array}$ & $\begin{array}{l}F_{R_{\mu}} \\
(\mathrm{dB})\end{array}$ & $\begin{array}{l}F_{R_{\sigma}} \\
(\mathrm{dB})\end{array}$ & & \\
\hline \multirow{4}{*}{ A } & I & -57.2 & 2696 & 9.93 & -1.94 & 2.54 & 282 & 31.7 & 8.13 & 6.25 & 168 & 4.06 & 2.62 & 0.57 & 3146 & 5627 \\
\hline & II & -48.9 & 2415 & 44.1 & 9.74 & 4.27 & 288 & 47.7 & 21.1 & 8.09 & 122 & 4.71 & 2.39 & 0.63 & 2825 & 8302 \\
\hline & III & -53.3 & 2673 & 42.7 & 1.19 & 4.08 & 504 & 43.1 & 9.65 & 5.66 & 278 & 0.20 & -3.06 & 1.39 & 3455 & 10827 \\
\hline & IV & -52.5 & 6516 & 39.8 & -1.67 & 4.20 & 898 & 41.0 & 1.99 & 4.90 & 246 & 2.64 & 0.50 & 0.69 & 7660 & 13659 \\
\hline \multirow{4}{*}{ B } & I & -29.9 & 334 & 1.34 & -0.44 & 0.69 & 265 & 10.2 & 4.91 & 2.52 & 391 & 1.96 & -0.49 & 0.65 & 990 & 8409 \\
\hline & II & -28.2 & 454 & 0.56 & -0.15 & 0.28 & 369 & 19.5 & 8.40 & 4.71 & 803 & 2.70 & 0.65 & 0.46 & 1626 & 10940 \\
\hline & III & -29.1 & 345 & 1.15 & 0.27 & 0.40 & 505 & 34.3 & 15.7 & 8.45 & 730 & 2.44 & -0.12 & 0.79 & 1553 & 8660 \\
\hline & IV & -29.2 & 841 & 10.5 & 0.31 & 1.56 & 1115 & 61.0 & 18.2 & 9.66 & 652 & 6.33 & 0.31 & 0.83 & 2608 & 11745 \\
\hline \multirow{4}{*}{ C } & I & -53.8 & 1154 & 3.80 & 0.44 & 0.75 & 272 & 9.16 & 4.33 & 2.63 & 1101 & 1.26 & -0.17 & 0.41 & 2527 & 6200 \\
\hline & II & -52.9 & 315 & 5.29 & 1.56 & 1.34 & 430 & 43.9 & 12.3 & 5.93 & 589 & 11.1 & 6.30 & 1.91 & 1334 & 4537 \\
\hline & III & -55.4 & 1093 & 3.54 & -1.77 & 1.11 & 314 & 45.9 & 6.28 & 5.59 & 342 & 0.17 & -2.60 & 0.84 & 1749 & 6461 \\
\hline & IV & -61.2 & 2090 & 32.3 & 0.27 & 3.33 & 632 & 38.7 & 11.1 & 5.73 & 3807 & 16.3 & -12.0 & 3.34 & 6529 & 15860 \\
\hline
\end{tabular}

\section{A. Both Persons Oriented Toward Oncoming Traffic (Scenario A)}

Across all channel measurements in this scenario, there was an observable trend to the disturbances caused by each of the vehicles. As shown in Table I, the disturbance duration for all vehicles during the approaching region in Scenario A was larger than those recorded for the other scenarios. This was presumably due to the fact that both person's bodies faced in the direction of the oncoming vehicles meaning that they were more prone to scattered and reflected signal contributions introduced by the movement of the vehicles. As an example, Fig. 6(a) shows the received signal power time series for the W2W channel when perturbed by a Type III vehicle. As the vehicle approached both people (points $P_{A}$ to $P_{T}$ ), it began to have an impact on the received signal power well before reaching the two people forming the W2W link [2673 ms (Table I)]. This value was comparable with those for the Type I and II vehicles, which were 2696 and $2415 \mathrm{~ms}$, respectively. However, the maximum fade experienced showed significant disparity, with the Type I vehicle inducing a much smaller maximum fade of $9.93 \mathrm{~dB}$ when compared with the Type II $(44.1 \mathrm{~dB})$ and Type III $(42.7 \mathrm{~dB})$ vehicles. These results suggest that small-to-medium sized vehicles can cause fluctuations in the channel for a W2W link from approximately the same distance when both bodies are oriented toward oncoming traffic; however, the magnitude of the disturbance depends on the vehicle size. Interestingly, although the Type IV vehicle disturbs the channel for significantly longer than the smaller vehicles $(6516 \mathrm{~ms})$ in the approaching region, the maximum fade experienced was observed to be comparable with the Type II and III vehicles.

For all vehicle types during the transitioning region, the channel was subject to significant fading (Table I) due to the vehicle obscuring the direct signal path between the two persons. This can be seen visually in Fig. 6(a) between points $P_{T}$ and $P_{R}$, where the blocking effect of the vehicle reduced the mean signal power significantly when compared with the approaching region. As expected, the duration of the shadowing event is directly related to the vehicle type, with
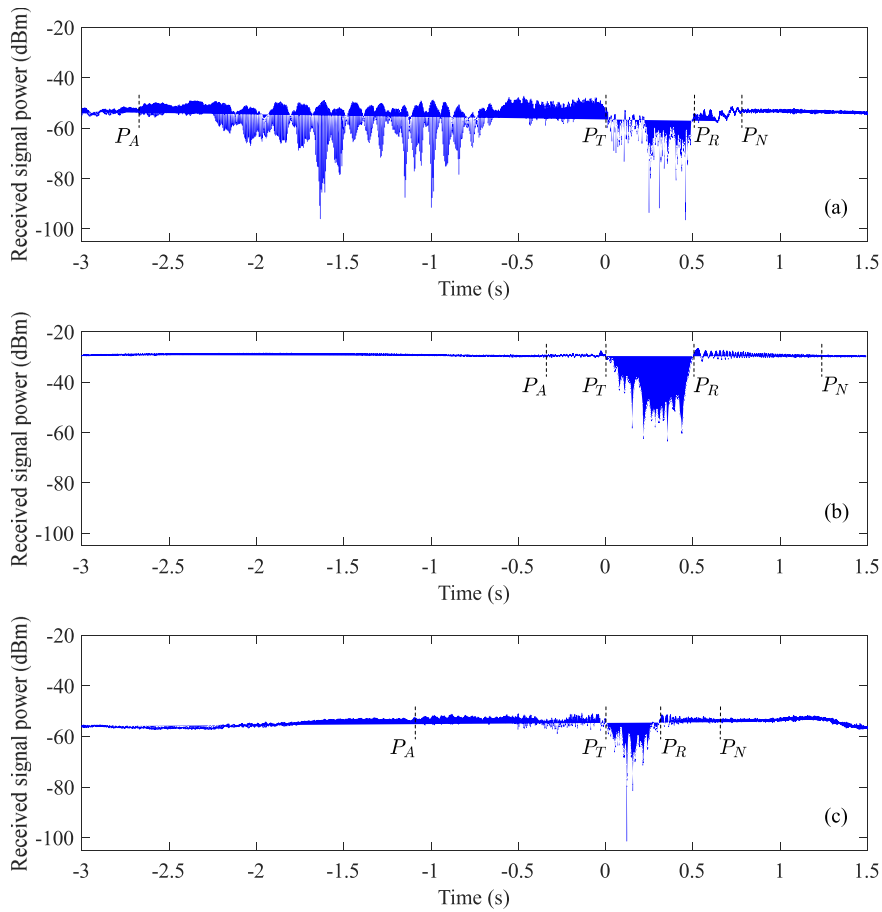

Fig. 6. Received signal power time series for the Type III vehicle in (a) Scenario A, (b) Scenario B, and (c) Scenario C. Points $P_{A}, P_{T}, P_{R}$, and $P_{N}$ mark the beginning of the approaching, transitioning, and receding regions and return to unperturbed state (i.e., end of disturbance), respectively.

larger vehicle sizes (i.e., Types III and IV) causing longer disturbances. Unique to this particular scenario, as both person's bodies were facing in the same direction, the impact on the $\mathrm{W} 2 \mathrm{~W}$ channel of the vehicle entering the receding region was greatly reduced compared with the other scenarios. This, of course, was to be expected, due to the bodies obstructing the majority of reflected waves emanating from the vehicle as it traversed this region. Furthermore, as shown between points $P_{R}$ and $P_{N}$ in Fig. 6(a) and Table I for the other vehicle types, the $\mathrm{W} 2 \mathrm{~W}$ channel was observed to return to its unperturbed state in a relatively short period of time when compared with 
the equivalent approaching segment. Nonetheless, there was no apparent correlation between the duration of the disturbance and vehicle type for this region of Scenario A.

\section{B. Both Persons Oriented Toward One Another (Scenario B)}

For brevity, we only discuss the impact of the Type III vehicle during Scenario B, however, a full summary of our results for other types of traffic is provided in Table I. When compared with Scenario A, it became immediately obvious that due to the much stronger LOS condition (evidenced by the increase in the received signal power [see Fig. 6(b) and Table I]), oncoming traffic only perturbed the W2W link when the vehicle was in its immediate vicinity. From Table I, the duration of disturbance as the Type III vehicle approached lasted for $345 \mathrm{~ms}$, which was considerably less than that obtained for the same region in Scenario A. However, in the transitioning region, the disturbance lasted for approximately $505 \mathrm{~ms}$, which was comparable with the same type of vehicle in the equivalent region during Scenario A. During the transitioning phase, the relative body orientation of the two persons meant that the vehicle-induced fades were not as deep compared with Scenario A. For example, the Type III vehicle here experienced a maximum fade of $34.3 \mathrm{~dB}$, which is notably lower than the value of $43.1 \mathrm{~dB}$ observed in Scenario A.

In Scenario B, the relative orientation of the two person's bodies meant that in the receding region the vehicles had a greater impact on the $\mathrm{W} 2 \mathrm{~W}$ channel. This was largely due to the fact that their bodies no longer fully shadowed the multipath signal contributions emanating from the direction of the vehicle. For example, the disturbance duration for a Type III vehicle was $730 \mathrm{~ms}$, which was more than twice the duration when compared with Scenario A. Interestingly, although the relative orientation of both persons should have made the W2W link equally susceptible to reflected signals emanating from the vehicle as it moved through both the approaching and the receding regions, the vehicle had a noticeably greater impact during the receding region. This was presumably due to the nonsymmetrical front and rear cross-sectional areas of the vehicle. In particular the dimensions of these cross-sectional areas, which tends to be smaller at the front for aerodynamics, would have likely reflected fewer signal components in the direction of person $\mathrm{B}$ than the relatively perpendicular rear section. As a result, the Type III vehicle generated stronger reflections in the receding region, evident from both the larger duration of disturbance and the maximum fade depth than the approaching region.

\section{Both Persons Oriented in the Opposite Directions (Scenario C)}

In contrast to Scenarios A and B, this situation investigated the compounded effects of vehicle-induced signal variations and shadowing caused by both person's bodies on the W2W link. As shown in Table I, the approaching region for the Type III vehicle lasted for $1093 \mathrm{~ms}$. Compared with the approaching section of Scenario A, a maximum fade of $3.54 \mathrm{~dB}$ was observed, which was significantly smaller than Scenario A $(42.7 \mathrm{~dB})$. It is evident from both of these metrics that the orientation of person B away from the oncoming vehicle significantly reduced its impact on the W2W channel within this region. In the transitioning region, the disturbance lasted for $314 \mathrm{~ms}$, which was shorter than Scenarios A and B, however, this could be accounted for due to a smaller Type III vehicle that was recorded for this particular scenario. This result, coupled with earlier observations from Scenarios A and B, suggests that the duration of disturbance within the transitioning region is largely independent of the relative orientation of the person's bodies and dependent, instead, on the length of vehicle obscuring the direct signal path. However, similar to Scenarios A and B, relative body orientation does have an impact on the receding phase of the vehicle's journey. In this case, the orientation of person B in the direction of the receding vehicle permitted an increased disturbance period of $342 \mathrm{~ms}$ for a Type III vehicle when compared with Scenario A [278 ms (Table I)]. Also, from Table I, the significance of relative body orientation on the receding region is particularly evident for the largest vehicle during Scenario C. Here, the disturbance for the Type IV vehicle was observed to last for $3807 \mathrm{~ms}$ and contained a maximum fade of $16.3 \mathrm{~dB}$, which was a significantly greater than the disturbance recorded for any other vehicle within this region.

\section{LONG-TERM FADING}

To extract the long-term fading from the received signal power, the signal fluctuations that occurred due to short-term fading must first be removed. In this paper, both persons who formed the $\mathrm{W} 2 \mathrm{~W}$ link remained relatively stationary, with any long-term variations in the channel largely caused by involuntary movements and other slow movements in the local surroundings. To determine an appropriate smoothing window for the approaching and receding regions, a number of windows were applied to the measurement data. It was deduced that a window size of $100 \mathrm{~ms}$ (or equivalently 1000 samples) most accurately captured the longterm fading behavior. During the transitioning region, as the vehicle intersected the direct LOS signal path between the W2W links, more frequent long-term variations occur caused by vehicle-induced shadowing as well as the usual shortterm fading. Therefore, for the transitioning case, a smaller, $10 \mathrm{~ms}$ (100 samples), window size was required due to the increased volatility of the W2W link. To further assist with the interpretation of our results, we also removed the transmit power from our data, so that all fade levels are now referenced from $0 \mathrm{dBm}$ meaning that they are synonymous with the attenuation.

Upon studying the form of the long-term fading component over each of the three distinct regions of the vehicle's journey, it became apparent that the empirical probability density functions (pdfs) contained a number of modes, presumably relating to the nonhomogeneous signal shadowing induced by the vehicle as it moved within the vicinity of the $\mathrm{W} 2 \mathrm{~W}$ channel. Therefore, a GMM was chosen to model the longterm fading. Mixture models assume a linear superposition of $N$ multivariate distributions and are often used where one statistical model does not accurately model a complete data 
set [24]-[26]. The pdf of a mixture model can be represented as $[27$, eq. (11.5)]

$$
f(x \mid \alpha, \theta)=\sum_{n=1}^{N} \alpha_{n} g_{n}\left(x \mid \theta_{n}\right)
$$

where $x$ is a vector of a random variable of length $y$, and $g_{n}\left(x \mid \theta_{n}\right)$ is the component density function with parameters represented by $\theta_{n}$. The weights of each density function are given by $\alpha_{n}$ with the constraint that they are nonnegative and must satisfy the normality condition $\sum_{n=1}^{N} \alpha_{n}=1$. These weights are also called the mixing proportions or mixing coefficients. The component densities can be any pdf, but in this paper, we will utilize the multivariate Gaussian density. The pdf for a multivariate Gaussian finite mixture is given by $[24$, eq. (2)]

$$
f(x \mid \alpha, \mu, \Sigma)=\sum_{n=1}^{N} \alpha_{n} \phi\left(x \mid \mu_{n}, \Sigma_{n}\right)
$$

where $\phi(\cdot)$ represents a multivariate Gaussian pdf, which, in a singular dimension form, is given by

$$
\phi\left(x \mid \mu_{n}, \Sigma_{n}\right)=\frac{1}{\sqrt{2 \pi} \sigma_{n}} \exp \left[-\frac{1}{2 \sigma_{n}^{2}}\left(x_{i}-\mu_{n}\right)^{2}\right]
$$

where $\mu_{n}$ denotes the mean, $\sigma_{n}^{2}$ denotes the variance, and $\Sigma_{n}$ denotes the covariance matrix of the $n$ components. For a GMM, if $n$ is known, then the estimation of the parameters can be achieved using the expectation-maximization (EM) algorithm [28].

The EM-algorithm proceeds iteratively in two steps. First, the expectation step treats the Gaussian parameters $\mu_{n}, \Sigma_{n}$, and $\alpha_{n}$ as fixed. Then, for each measured data point, the probability that it is contained within each cluster $n$ is evaluated using the responsibility value. This gives a soft membership for each data point to the cluster $n$, which contains the highest responsibility value, i.e., has the greatest probability of belonging to that cluster. The second step, the maximization step, treats the soft assignments of the data points to each cluster as fixed, and updates the Gaussian parameters using an estimate weighted by the probability of the responsibility value. These steps are iteratively performed, thereby increasing the loglikelihood of the GMM and hence converging to a finite solution. However, in practice, the iterative procedure is stopped after a predefined threshold in satisfied. In this paper, all parameter estimates were obtained using the gmaistribution function available in the Curve Fitting Toolbox of MATLAB. Since the data are nonconvex in form, many local minima are possible. Thus, to improve the likelihood of a global optimal solution, the starting position for the cluster centers was estimated using the $k$-means clustering technique, which partitions the data into $n$ groups, such that the within-group sum of squares is minimized [27]. This was achieved using the kmeans function also available in MATLAB.

Intuitively, the more Gaussian pdfs that are used to model the underlying data, the better the model fit. However, increasing the number of Gaussian distributions also acts to increase the model complexity. Therefore, the number of Gaussian densities required to model the data in this paper was selected
TABLE II

AIC Estimates For Approaching Region In SCEnARio A

\begin{tabular}{cccccc}
\hline \hline Vehicle & \multicolumn{5}{c}{ Gaussian Model Order $(N)$} \\
Type & 1 & 2 & 3 & 4 & 5 \\
\hline I & 486 & 477 & 475 & 477 & 483 \\
II & 596 & 587 & 585 & 587 & 593 \\
III & 481 & 487 & 470 & 472 & 478 \\
IV & 457 & 448 & 446 & 452 & 453 \\
\hline \hline
\end{tabular}

using a complexity penalty analysis, in this case the Akaike information criterion (AIC) [29], [30]. The AIC is defined as [30, eq. (3.44)]

$$
\mathrm{AIC}=-2 L(\hat{\theta} \mid x)+2 p
$$

where $L(\hat{\theta} \mid x)$ is the maximized log likelihood over the unknown parameters $\theta$ given the data and the model, and $p$ is the number of estimated parameters for the model under test. The AIC can be easily computed, since the log likelihood is readily available from the EM estimates. In (4), the first term indicates the model fit to the data, where the lowest log likelihood yields the model with minimal estimation losses. The second term penalizes the use of additional parameters, ensuring that overfitting of the model does not occur [30]. As noted by Fort et al. [31], the AIC gives a relative measure of the goodness of fit from the models available, but it does not guarantee that the chosen model provides an adequate fit to the data. Therefore, in this paper, all candidate models were compared visually with the empirical data to ensure that a satisfactory fit was obtained.

\section{A. Both Persons Oriented Toward Oncoming Traffic (Scenario A)}

As an example of the model selection process, the results of the AIC computations for each vehicle type in the approaching region for increasing numbers of Gaussian densities are provided in Table II. It was found that the minimum AIC value was continually obtained for a mixture model containing three Gaussian distributions. Although a third-order GMM provided only a marginally improved fit over a second-order fit in some cases, to simplify the analysis and to enable a direct comparison between vehicle types, we persist with a thirdorder GMM throughout this paper. In the approaching region with both person's facing in the direction of the oncoming vehicle, a long-term variation of the signal power in the $\mathrm{W} 2 \mathrm{~W}$ channel was found to occur, which was not evident during the unperturbed channel state. If we consider the earlier example of the received signal power given in Fig. 6(a) as a Type III vehicle approached the two persons, long-term variation of the signal power is clearly evident between points $P_{A}$ and $P_{T}$. As shown in Table III, this led to three clustered long-term fading levels at $-74.2,-72.7$, and $-69.6 \mathrm{~dB}$ albeit with small the standard deviations of $0.1,0.2$, and $0.6 \mathrm{~dB}$, respectively. This observation coupled with the results for the Type I, II, and IV vehicles (Table III) suggests that for a W2W link when both nodes face in the direction of an approaching vehicle, noticeable long-term signal variations can occur, which may be directly attributed to the presence of the vehicle. 
TABLE III

GMM PARAMETERS FOR ALL SCENARIOS

\begin{tabular}{|c|c|c|c|c|c|c|c|c|c|c|c|}
\hline \multirow{2}{*}{ Scenario } & \multirow{2}{*}{ Region } & \multirow{2}{*}{$\begin{array}{l}\text { Vehicle } \\
\text { Type }\end{array}$} & \multicolumn{3}{|c|}{$n=1$} & \multicolumn{3}{|c|}{$n=2$} & \multicolumn{3}{|c|}{$n=3$} \\
\hline & & & $\alpha_{1}$ & $\mu_{1}$ & $\sigma_{1}$ & $\alpha_{2}$ & $\mu_{2}$ & $\sigma_{2}$ & $\alpha_{3}$ & $\mu_{3}$ & $\sigma_{3}$ \\
\hline \multirow{8}{*}{ A } & \multirow{4}{*}{ Approaching } & I & 0.40 & -74.8 & 0.80 & 0.20 & -72.2 & 0.10 & 0.40 & -70.7 & 0.50 \\
\hline & & II & 0.20 & -80.4 & 0.70 & 0.10 & -78.2 & 0.20 & 0.70 & -74.2 & 2.70 \\
\hline & & III & 0.20 & -74.2 & 0.10 & 0.40 & -72.7 & 0.20 & 0.40 & -69.6 & 0.60 \\
\hline & & IV & 0.20 & -69.9 & 0.40 & 0.60 & -67.8 & 0.50 & 0.20 & -66.3 & 0.10 \\
\hline & \multirow{4}{*}{ Transitioning } & I & 0.10 & -93.2 & 3.30 & 0.50 & -85.2 & 8.40 & 0.40 & -77.4 & 4.50 \\
\hline & & II & 0.20 & -96.7 & 6.80 & 0.60 & -86.5 & 15.5 & 0.20 & -76.4 & 0.60 \\
\hline & & III & 0.10 & -88.2 & 0.60 & 0.40 & -82.8 & 5.70 & 0.50 & -76.4 & 4.00 \\
\hline & & IV & 0.10 & -75.6 & 5.70 & 0.50 & -72.3 & 2.50 & 0.40 & -69.5 & 2.40 \\
\hline \multirow{4}{*}{ B } & \multirow{4}{*}{ Transitioning } & I & 0.50 & -54.5 & 1.20 & 0.20 & -52.0 & 0.10 & 0.30 & -49.9 & 3.20 \\
\hline & & II & 0.60 & -56.9 & 10.7 & 0.10 & -53.5 & 0.20 & 0.30 & -48.3 & 1.60 \\
\hline & & III & 0.50 & -68.9 & 12.9 & 0.40 & -56.8 & 10.5 & 0.10 & -48.6 & 0.60 \\
\hline & & IV & 0.30 & -73.1 & 13.8 & 0.40 & -63.3 & 7.90 & 0.30 & -52.6 & 8.70 \\
\hline \multirow{4}{*}{$\mathrm{C}$} & \multirow{4}{*}{ Transitioning } & I & 0.70 & -77.1 & 1.00 & 0.10 & -73.4 & 0.20 & 0.20 & -71.1 & 1.00 \\
\hline & & II & 0.10 & -96.5 & 2.00 & 0.60 & -83.4 & 12.6 & 0.30 & -78.3 & 1.80 \\
\hline & & III & 0.30 & -83.7 & 15.1 & 0.30 & -78.9 & 2.20 & 0.40 & -74.1 & 1.70 \\
\hline & & IV & 0.10 & -97.2 & 1.20 & 0.20 & -92.2 & 1.30 & 0.70 & -88.0 & 8.90 \\
\hline
\end{tabular}
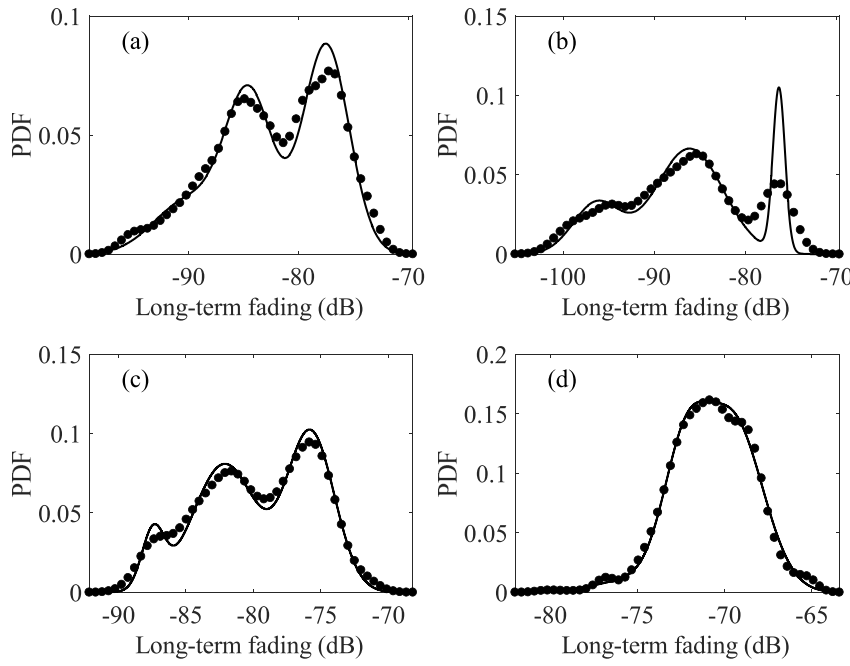

Fig. 7. PDFs of the empirical long-term fading (circle shapes) and GMM (continuous lines) for (a) Type I, (b) Type II, (c) Type III, and (d) Type IV vehicles during the transitioning region for Scenario A. The estimated model parameters are provided in Table III.

As the vehicles traversed into the transitioning region, the long-term fading was subject to significant vehicle-induced shadowing caused by the obscuration of the direct signal path. The substantial impact of the vehicle on the W2W channel in the transitioning region can be seen in Table III. Here, the mean values for the clustered long-term fading levels were significantly reduced, while the standard deviations for all modes of the GMMs were observed to increase significantly compared with the approaching region. Fig. 7 shows the empirical and estimated theoretical pdfs for each of the four vehicles shown in Fig. 4. Considering the Type I vehicle shown in Fig. 4(a), the corresponding long-term fading distribution contained three fading levels clustered around -93.2, -85.2, and $-77.4 \mathrm{~dB}$, as shown in Fig. 7(a). The greatest degradation occurred when the tallest section of the vehicle obscured the channel. In this case, the tallest portion of the vehicle only accounted for a small cross section of the total vehicle length. This is evident from Table III, since the cluster that contains the most significant reduction of the long-term fading only accounts for 0.1 of the total mixing proportion for the GMM. For comparison, Fig. 7(b) shows the empirical and estimated pdfs for the larger dimensioned Type II vehicle, which caused an even greater degradation in the long-term fading with an increased mixing proportion for the equivalent signal cluster (Table III).

Inspection of Fig. 7(c) clearly indicates that three grouped long-term fading levels were also present for the Type III vehicle. As shown in Fig. 4(c), this vehicle can be identified as a small bus with a large proportion of glass windows along its sides. As a result, the direct signal path actually suffered less attenuation compared with the Type I and II vehicles (Table III). The empirical pdf for the Type IV vehicle also contained three clusters, but in comparison with the other vehicle types, the peaks are not as distinguishable [Fig. 7(d)]. In this instance, the Type IV vehicle was a large lorry [Fig. 4(d)]. A close examination of Fig. 4(d) shows a large gap under the trailer and fabric sidewalls, which would offer less attenuation to the impinging electromagnetic waves than the metallic structures of the Type I and II vehicles. As the vehicle entered the receding region, the relative body orientations of the two persons limited the vehicular impact on the long-term fading, owing to the wearer's bodies shadowing the majority of the signal arriving from the direction of the receding vehicle. Therefore, it was determined that only the approaching and transitioning regions had sufficient variation in the long-term fading to merit an in-depth characterization.

\section{B. Both Persons Oriented Toward One Another (Scenario B)}

In this scenario (and Scenario C), the alignment of the two persons resulted in significantly less variation in the longterm fading when the vehicles moved through the approaching 

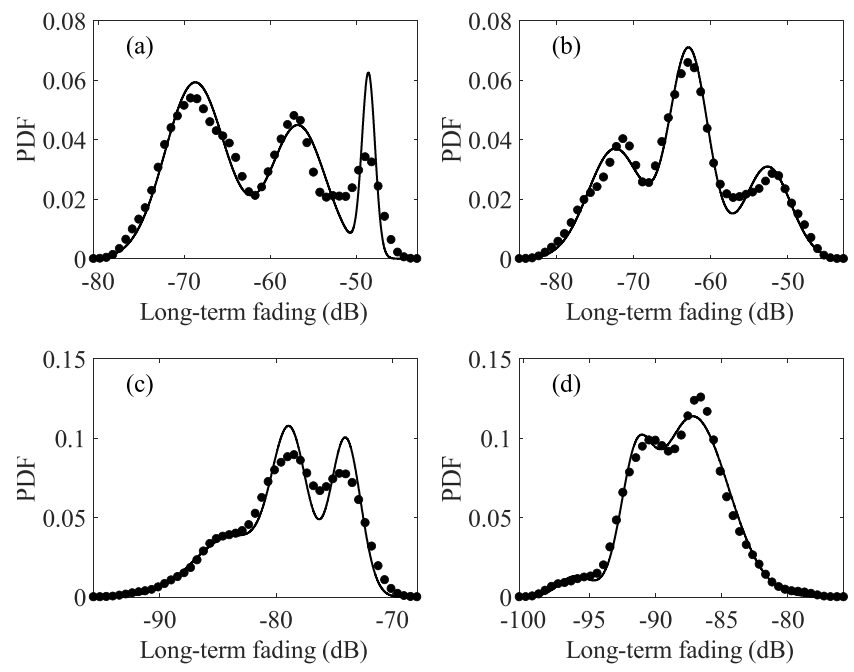

Fig. 8. PDFs of the empirical long-term fading (circle shapes) and GMM (continuous lines) for (a) Type III and (b) Type IV vehicles in Scenario B and (c) Type III and (d) Type IV vehicles in Scenario C in the transitioning region. The estimated model parameters are provided in Table III.

and receding regions. Therefore, in a similar fashion to the receding region of Scenario A, these cases were deemed not to be worthy of an in-depth characterization (particularly for the smaller vehicle types). Therefore, we limit ourselves to a characterization of the long-term fading in the transitioning region only for Scenario B (and later for Scenario C).

As an example, Fig. 8(a) and (b) shows the empirical pdfs of the long-term fading for the Type III and IV vehicles, respectively. For the Type III vehicle [Fig. 8(a)], three distinct long-term fading levels can be identified with the majority of fading occurring within the first and second modes. In this instance, the dominant mode contained the most significant long-term fading, clustered around the $-68.9 \mathrm{~dB}$ level. Likewise, the shadowing induced in the long-term fading by the Type IV vehicle also exhibited three distinct fading groupings, each containing significant signal variability (as evidenced by the large standard deviations recorded for each cluster). Interestingly, despite the larger overall dimensions of the Type IV vehicle, its impact on the long-term fading of the W2W channel [Fig. 8(b)] was less pronounced than the Type III vehicle. Similar to the Type IV vehicle in Scenario $\mathrm{A}$, it is likely that the space below the truck permitted some signal contributions to reflect off the road surface underneath the trailer, reducing the severity of shadowing caused by this vehicle type. It should be noted that the Type IV vehicle considered for Scenario B had a trailer constructed entirely from metal, which accounted for the increased shadowing when compared with Scenario A.

\section{Both Persons Oriented in the Opposite Directions (Scenario C)}

In this scenario, both bodies were orientated so that they were parallel to the roadside and facing in the opposite directions to one another, resulting in a partial body shadowing situation similar to that observed in Scenario A. As an example of the long-term fading characteristics obtained in this scenario, Fig. 8(c) and (d) shows the empirical pdfs for the
Type III and IV vehicles, respectively, as they moved through the transitioning region. Again, for the Type III vehicle, three long-term fading groupings were prominent in the empirical pdf. Consulting Table III, it is evident that while all three fading clusters occurred with comparable mixing probabilities, the first cluster (centered around $-83.7 \mathrm{~dB}$ ) had by far the largest spread in values $(15.1 \mathrm{~dB})$, larger than any other cluster contained within Table III. Nonetheless, it was the presence of the Type IV vehicle in the transitioning region during Scenario $\mathrm{C}$, which caused the lowest overall long-term fading levels [Fig. 8(d)]. Here, the long-term fading was clustered around the $-97.2,-92.2$, and $-88 \mathrm{~dB}$ levels.

\section{SHORT-TERM FADING}

At the outset, it was anticipated that nearby vehicular traffic would cause substantial fluctuations in the short-term fading observed in W2W channels, induced mainly by the vehicles highly reflective (metallic) exterior, their nonuniform shapes and sizes, and relatively high velocity of travel. In the sequel, to ascertain the statistical characteristics of the short-term fading in relation to vehicle type and relative user orientation, an initial investigation was undertaken using the $\kappa-\mu$ distribution, which was chosen for its versatility and well-known ability to account for many of the popular fading models used in the literature (e.g., Nakagami- $m$, Rice, and Rayleigh) [32]. The $\kappa-\mu$ distribution intuitively comprises of two fading parameters, $\kappa>0$, which is simply the ratio of the total power of the dominant components $\left(d^{2}\right)$ to the total power of the scattered waves $\left(2 \mu \sigma^{2}\right)$, and $\mu>0$, which is related to the multipath clustering of the scattered waves [33]. The pdf, $f_{R}(r)$, of a fading signal envelope, $R$, which undergoes $\kappa-\mu$ fading, is given as [32, eq. (11)]

$$
\begin{aligned}
f_{R}(r)= & \frac{2 \mu(1+\kappa)^{\frac{\mu+1}{2}}}{\bar{r} \kappa^{\frac{\mu-1}{2}} \exp (\mu \kappa)}\left(\frac{r}{\bar{r}}\right)^{\mu} \\
& \times \exp \left[-\mu(1+\kappa)\left(\frac{r}{\bar{r}}\right)^{2}\right] I_{\mu-1}\left[2 \mu \sqrt{\kappa(1+\kappa)} \frac{r}{\bar{r}}\right]
\end{aligned}
$$

where $\bar{r}^{2}=E\left[R^{2}\right]$ is the mean signal power [with $E(\cdot)$ denoting the expectation operator], $\bar{r}^{2}=d^{2}+2 \mu \sigma^{2}$, and $I_{v}(\cdot)$ is the modified Bessel function of the first kind and order $v$. Nonetheless, throughout the course of the characterization work, it became increasingly apparent that the W2W channel in an urban environment often underwent severe fading [34]. Therefore, the $\kappa-\mu$ extreme distribution, which was first proposed in [32] and later detailed in [35] and [36] as an extension to the $\kappa-\mu$ distribution, was included in the set of candidate fading models considered in this paper. To obtain the $\kappa-\mu$ extreme distribution from the $\kappa-\mu$ distribution, the following relationship between $\kappa, \mu$, and the Nakagami $m$ parameter is used [35, eq. (2)]

$$
m=\frac{\mu(1+\kappa)^{2}}{1+2 \kappa} \text {. }
$$

By keeping $m$ constant and allowing the $\kappa$ and $\mu$ parameters to assume extreme values, i.e., $\kappa \rightarrow \infty$ (indicating a very strong LOS or dominant signal component) and $\mu \rightarrow 0$ 
TABLE IV

$\kappa-\mu$ AND $\kappa-\mu$ EXTREme PARAmeter Estimates FOR All ScEnARios

\begin{tabular}{|c|c|c|c|c|c|c|c|c|c|c|c|c|c|c|c|c|}
\hline \multirow{3}{*}{ Region } & \multirow{3}{*}{$\begin{array}{l}\text { Vehicle } \\
\text { Type }\end{array}$} & \multicolumn{5}{|c|}{ Scenario A } & \multicolumn{5}{|c|}{ Scenario B } & \multicolumn{5}{|c|}{ Scenario C } \\
\hline & & \multicolumn{3}{|c|}{$\kappa-\mu$} & \multicolumn{2}{|c|}{$\kappa-\mu$ Extreme } & \multicolumn{3}{|c|}{$\kappa-\mu$} & \multicolumn{2}{|c|}{$\kappa-\mu$ Extreme } & \multicolumn{3}{|c|}{$\kappa-\mu$} & \multicolumn{2}{|c|}{$\kappa-\mu$ Extreme } \\
\hline & & $\hat{\kappa}$ & $\hat{\mu}$ & $R A D$ & $\hat{m}$ & $R A D$ & $\hat{\kappa}$ & $\hat{\mu}$ & $R A D$ & $\hat{m}$ & $R A D$ & $\hat{\kappa}$ & $\hat{\mu}$ & $R A D$ & $\hat{m}$ & $R A D$ \\
\hline \multirow{4}{*}{ Approaching } & I & $\infty$ & 0.02 & 0.014678 & 12.0 & 0.014675 & $\infty$ & 0.01 & 0.022267 & 54.6 & 0.022268 & $\infty$ & 0.06 & 0.001088 & 115 & 0.001088 \\
\hline & II & 870 & 0.01 & 0.033228 & 5.83 & 0.033082 & $\infty$ & 0.07 & 0.022939 & 253 & 0.003666 & $\infty$ & 0.02 & 0.008352 & 14.1 & 0.008354 \\
\hline & III & 680 & 0.01 & 0.006391 & 3.42 & 0.006422 & $\infty$ & 0.01 & 0.010381 & 153 & 0.010883 & $\infty$ & 0.01 & 0.003276 & 23.1 & 0.003276 \\
\hline & IV & 550 & 0.01 & 0.011476 & 2.81 & 0.011612 & $\infty$ & 0.04 & 0.189461 & 63.5 & 0.189499 & $\infty$ & 0.01 & 0.019160 & 4.78 & 0.019136 \\
\hline \multirow{4}{*}{ Transitioning } & I & $\infty$ & 0.02 & 0.084571 & 16.8 & 0.084559 & $\infty$ & 0.03 & 0.009790 & 220 & 0.001348 & $\infty$ & 0.06 & 0.005114 & 173 & 0.002791 \\
\hline & II & $\infty$ & 0.01 & 0.039196 & 5.51 & 0.039107 & $\infty$ & 0.09 & 0.032516 & 253 & 0.011948 & $\infty$ & 0.02 & 0.101092 & 28.5 & 0.101091 \\
\hline & III & 900 & 0.01 & 0.024042 & 4.61 & 0.023994 & $\infty$ & 0.01 & 0.110814 & 111 & 0.110813 & $\infty$ & 0.01 & 0.056379 & 24.3 & 0.056378 \\
\hline & IV & 560 & 0.01 & 0.041424 & 2.79 & 0.041371 & 830 & 0.01 & 0.029593 & 3.75 & 0.029704 & 310 & 0.01 & 0.011276 & 1.73 & 0.011022 \\
\hline \multirow{4}{*}{ Receding } & I & $\infty$ & 0.01 & 0.004666 & 72.5 & 0.004665 & $\infty$ & 0.01 & 0.043759 & 88.5 & 0.043758 & $\infty$ & 0.04 & 0.001260 & 137 & 0.001260 \\
\hline & II & $\infty$ & 0.01 & 0.037151 & 87.2 & 0.037149 & $\infty$ & 0.02 & 0.025442 & 192 & 0.035478 & $\infty$ & 0.01 & 0.015236 & 17.0 & 0.015236 \\
\hline & III & $\infty$ & 0.02 & 0.011202 & 13.0 & 0.011202 & $\infty$ & 0.01 & 0.023448 & 54.2 & 0.023450 & $\infty$ & 0.01 & 0.030924 & 46.1 & 0.030921 \\
\hline & IV & $\infty$ & 0.01 & 0.008138 & 52.0 & 0.008138 & $\infty$ & 0.01 & 0.102963 & 131 & 0.102963 & $\infty$ & 0.01 & 0.009156 & 5.05 & 0.009151 \\
\hline
\end{tabular}

Note: The $\hat{\bar{r}}$ parameter was approximately unity in all cases and therefore omitted for clarity.

(indicating very few multipaths), then with some mathematical manipulation, the pdf, $f_{R}(r)$, of a $\kappa-\mu$ extreme fading signal envelope, $R$, can be expressed as [35, eq. (4)]

$$
f_{R}(r)=\frac{4 m I_{1}\left(4 m \frac{r}{\bar{r}}\right)}{\bar{r} \exp \left[2 m\left(1+\left(\frac{r}{\bar{r}}\right)^{2}\right)\right]}+\exp (-2 m) \delta\left(\frac{r}{\bar{r}}\right)
$$

where $\delta(\cdot)$ is the Dirac delta function. The short-term fading envelopes were obtained by removing the long-term fading variations calculated in Section $\mathrm{V}$ from the original received signal power time series, and then converting the result into a linear amplitude. All parameter estimates for the pdfs of the two candidate fading models were obtained using the lsqnonlin function with the multistart class to ensure that a global optimal solution was found, both available from the Optimization toolbox of MATLAB.

To assess the goodness of fit, we employed the resistoraverage distance $(R A D)$ [37], which is based on the Kullback-Leibler distance $\left(D_{\mathrm{KL}}\right)$ [38]. $D_{\mathrm{KL}}$ measures the relative entropy, or average information loss, between two pdfs by quantifying how well a particular pdf, $p(x)$, describes samples from another pdf, $q(x)$. Thus, letting $p(x)$ represent the empirical pdf and $q(x)$ denote either of the theoretical pdfs of the two candidate models, then the lower $D_{\mathrm{KL}}$ value indicates a closer approximation to the measured data. This can be further improved by calculating the harmonic mean between $D_{\mathrm{KL}}(p \| q)$ and $D_{\mathrm{KL}}(q \| p)$, and hence provide the symmetrical distance metric, $R A D$. This was calculated using $R A D(p, q)=\left(\left(D_{\mathrm{KL}}(p \| q)\right)^{-1}+\left(D_{\mathrm{KL}}(q \| p)\right)^{-1}\right)^{-1}$, where $D_{\mathrm{KL}}(p \| q)=\int_{-\infty}^{\infty} p(x) \log p(x) / q(x) d x$ and $D_{\mathrm{KL}}(q \| p)=$ $\int_{-\infty}^{\infty} q(x) \log q(x) / p(x) d x$. It should be noted that $\log (\cdot)$ here refers to the natural logarithm. Table IV provides the estimated parameters for the $\kappa-\mu$ and the $\kappa-\mu$ extreme pdfs, alongside the $R A D$ values indicating the goodness of fit for all scenarios.

\section{A. Both Persons Oriented Toward Oncoming Traffic (Scenario A)}

From Table IV, the severity of the fading can be immediately recognized from the $\kappa$ values, which were much greater than 500, and the $\mu$ values, which approached zero. As both bodies were oriented toward the oncoming traffic, the link was particularly susceptible to a significant signal contribution returned in the direction of the $\mathrm{W} 2 \mathrm{~W}$ link from the oncoming vehicle, irrespective of its type. This dominant component acted to constructively and destructively interfere with the signal contributions arriving from the direct signal path and the ground reflection associated with the direct path. As the estimated $\mu$ parameter approached zero, this suggested that no measureable scattered multipath contribution was present, possibly because it extended below the sensitivity of the receiver. The net result of this, i.e., no detectable scattered multipath contribution and a low number of dominant signal paths, makes a strong case for the use of the $\kappa-\mu$ extreme distribution [36] in this scenario.

As shown in Table IV, the RAD indicates that the $\kappa-\mu$ extreme distribution outperformed the $\kappa-\mu$ distribution in the majority of model fits to the empirical data. Nonetheless, for the remaining channels, the estimated $\kappa$ and $\mu$ parameters still approached extreme values suggesting that the $\kappa-\mu$ extreme fading model was suitable regardless of the vehicle type. Additionally, the estimated $m$ parameter of the $\kappa-\mu$ extreme distribution was found to decrease as the size of the approaching vehicle increased, suggesting that an increase in the severity of the short-term fading occurred.

As the vehicles traversed the transitioning region, the previously dominant LOS and ground reflected components between the two persons were now partially or fully obstructed by the passing vehicle depending on its size and shape. Similar to the approaching stage, the estimated $m$ parameters were found to reduce as the vehicle size increased. As an example of the model fitting, Fig. 9 shows a selection of the empirical pdfs compared with the pdf of the $\kappa-\mu$ extreme distribution. In particular, Fig. 9(a) and (b) shows the distribution of the short-term fading for the smallest (Type I) and largest (Type IV) vehicles as they approached the W2W channel. As we can see, the presence of the Type IV vehicle [Fig. 9(b)] caused much deeper fading in the W2W channel 

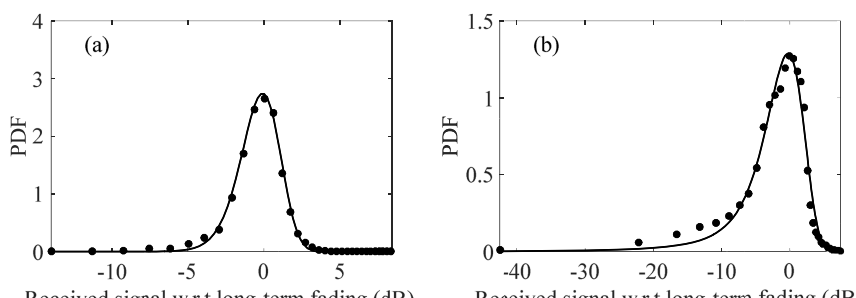

Received signal w.r.t long-term fading $(\mathrm{dB})$
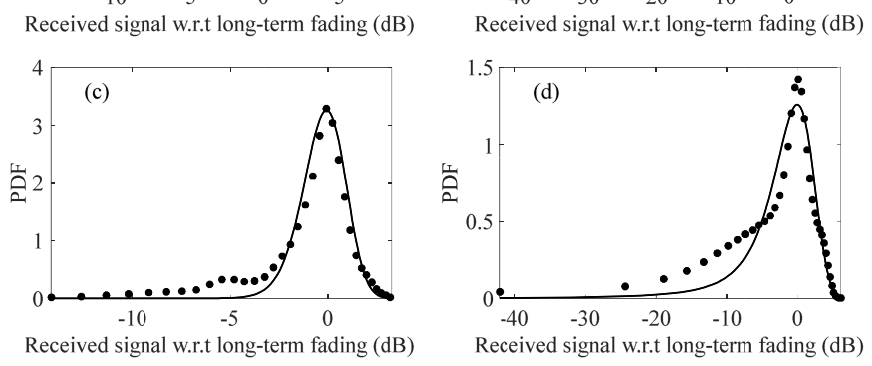

Fig. 9. PDFs of the empirical short-term fading with respect to the long-term fading (circle shapes) and $\kappa-\mu$ extreme (continuous lines) in Scenario A for (a) Type I and (b) Type IV vehicles for the approaching region and (c) Type I and (d) Type IV for the transitioning region. The estimated model parameters are provided in Table IV.

compared with the Type I automobile [Fig. 9(a)]. In contrast, Fig. 9(c) and (d) shows the same vehicle types within the transitioning region. Interestingly, the $m$ parameter estimates in Table IV suggest a similarity between the severity of fading experienced in the approaching and transitioning region in relation to vehicle types, which can be confirmed from the comparable shapes of the short-term fading distributions in Fig. 9(c) and (d).

In the transitioning region, the $R A D$ values indicated that the $\kappa-\mu$ extreme distribution provided a better fit to the underlying channel data than the $\kappa-\mu$ distribution for all vehicle types. As the vehicle progressed into the receding region, the effect of body shadowing acted to shield the W2W link from the vehicles. This resulted in no discernible impact on the channel from the smaller vehicle types and minimal short-term fading created by the larger vehicle types.

\section{B. Both Persons Oriented Toward One Another (Scenario B)}

The significance of the relative body orientation on the short-term signal variation became immediately obvious when both persons were orientated so that they were now directly facing one another. In this scenario, the majority of the estimated $\kappa$ parameters tended toward infinity ${ }^{2}$ while correspondingly, the estimated $m$ parameters of the $\kappa-\mu$ extreme distribution (Table IV) were also significantly increased compared with Scenario A. During the approaching region of Scenario B, since both persons were no longer directly facing oncoming traffic, their bodies partially shielded the electromagnetic waves emanating from the direction of the approaching vehicle, which resulted in a reduction of the shortterm fading caused by multipath interference.

In the transitioning region, the improved LOS conditions compared with Scenario A were found to mitigate the severity of the fading, particularly for the small and mid to large

\footnotetext{
${ }^{2}$ In this paper, we denote any estimated $\kappa$ parameter, for which $\kappa>1000$ as $\kappa \rightarrow \infty$
}
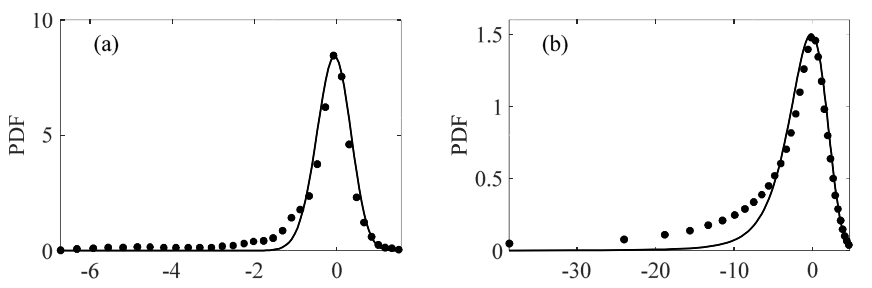

Received signal w.r.t long-term fading (dB)
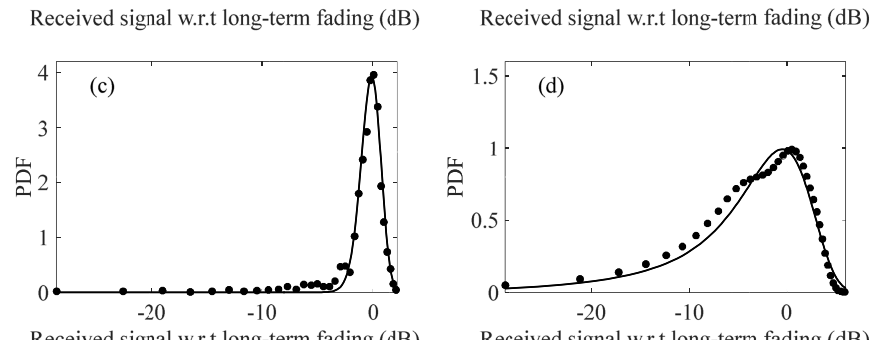

Fig. 10. PDFs of the empirical short-term fading with respect to the long-term fading (circle shapes) and $\kappa-\mu$ extreme (continuous lines) for (a) Type III and (b) Type IV vehicles for Scenario B and (c) Type III and (d) Type IV vehicles for Scenario $\mathrm{C}$ in the transitioning region. The estimated model parameters are provided in Table IV.

sized vehicles types [i.e., $m>100$ for the Type I-III vehicles (Table IV)]. On the other hand, the largest vehicle type was found to suffer from increased short-term fading within this region, likely due to its increased height and length (i.e., greater obscuration of the LOS and for longer durations) as it traversed through the direct signal path. As an example of the model fitting for this region, Fig. 10(a) and (b) presents the empirical pdfs of the short-term fading compared with their respective $\kappa-\mu$ extreme pdf fits for the Types III and IV vehicles. As we can see, the Type III vehicle underwent largely insignificant short-term fading [Fig. 10(a)] with the majority of the short-term signal distributed with $\pm 1 \mathrm{~dB}$ of the long-term fading level. In contrast, the Type IV vehicle experienced much greater short-term fading with fades greater than $20 \mathrm{~dB}$ below the long-term fading level occasionally possible [Fig. 10(b)].

As the vehicles traveled away from the two persons (i.e., receded), the propagation geometry was similar to that found in the approaching region for this scenario. Again, both person's bodies acted to partially shield the W2W link from signal contributions arriving from the direction of the retreating vehicle, which resulted in very little short-term fading, as evidenced by the large $m$ values obtained for this region. Again, the $R A D$ values indicated the majority of channels were modeled better using the $\kappa-\mu$ extreme distribution (Table IV).

\section{Both Persons Oriented in the Opposite Directions (Scenario C)}

In this scenario, at least one person was always facing in the direction of the moving vehicle, and thus, at least one end of the W2W link was continually exposed to the additional multipath. From Table IV, it can be seen that this acted to mitigate the severity of the short-term fading compared with the approaching and transitioning stages of Scenario A. Performing a like for like comparison, the estimated $m$ parameters obtained for these two regions of Scenario $\mathrm{C}$ were much greater than the corresponding values obtained for Scenario A (with the exception of the Type IV vehicle during the transitioning phase of Scenario C). 
In the receding region of Scenario $\mathrm{C}$, the W2W link was found to suffer less short-term fading effects during the presence of vehicle Types I-III than for the Type IV vehicle. In fact, the estimated $m$ value obtained for Scenario C [5.05 (Table IV)] for this region was the lowest across all three scenarios. As an example of the model fitting for this scenario, Fig. 10(c) and (d) shows the empirical short-term fading and $\kappa-\mu$ extreme pdfs for the Type III and IV vehicles within the transitioning region. Similar to the Type III vehicle in Scenario B [Fig. 10(a)], the majority of the short-term fading occurs close to the long-term fading level (i.e, within $\pm 2 \mathrm{~dB}$ ). Nonetheless, very rarely, fades beyond $5 \mathrm{~dB}$ below this level can occur [Fig. 10(c)]. As with this region in the other scenarios, the Type IV vehicle was observed to cause significant disturbance to the short-term fading [Fig. 10(d)]. Here we can see that short-term down fades greater than $20 \mathrm{~dB}$ occasionally occur. In a similar fashion to the other two scenarios, the $\kappa-\mu$ extreme distribution outperformed the $\kappa-\mu$ distribution, providing lower $R A D$ values for the majority of the cases considered in this scenario (Table IV).

\section{CONCLUSION}

In this paper, we have investigated the effect of vehicular traffic on W2W communications channels operating at $2.45 \mathrm{GHz}$ in an urban environment. In particular, we have investigated the statistical characteristics of the signal fading observed in a W2W link formed by two persons positioned on the opposite sides of a road. Based on a segmentation of our measured channel data, according to the vehicle size and position relative to the $\mathrm{W} 2 \mathrm{~W}$ link, we have found that when both persons face in the direction of the oncoming traffic, the channel was subject to significant disturbances. Fades of up to $44.1 \mathrm{~dB}$ (Type II) from the unperturbed state are possible, with vehicle-induced signal variations appearing as early as $6516 \mathrm{~ms}$ (Type IV) prior to the vehicle crossing the direct signal path of the W2W link. However, when one or both persons were oriented so that they faced away from the direction of the oncoming vehicle (Scenarios B and C), the susceptibility to fading caused by an approaching vehicle was observed to decrease significantly. When the vehicles traversed the direct signal path between the two persons (i.e., transitioning region), the duration of disturbance was observed to be largely independent of relative body orientation, dependent instead on the length of vehicle obscuring the LOS, while for a receding vehicle, the W2W link was noticeably influenced by the relative orientations of the two persons. In the scenarios where both bodies shielded or partially obscured the multipath signal originating from the direction of the retreating vehicle, the impact of the vehicle on the $\mathrm{W} 2 \mathrm{~W}$ channel was greatly reduced as evidenced by lower maximum fades and duration of disturbances obtained.

To further study the propagation mechanisms responsible for shaping the received signal power, we decomposed the signal power into its long- and short-term fading components. While not all of the regions suffered equally from the effects of long-term fading, most notable was the impact of the vehicle within the transitioning region for each of the scenarios. Due to vehicle-induced shadowing, the long-term fading process was found to be multimodal and well described by a GMM. To assess the model order, we employed the AIC and found that a third-order GMM was best suited to modeling the longterm fading. To model the short-term fading, we initially used the $\kappa-\mu$ distribution, however, an inspection of the estimated model parameters revealed that the $\mathrm{W} 2 \mathrm{~W}$ channel in an urban environment often underwent severe fading (i.e., $\kappa \rightarrow \infty$ and $\mu \rightarrow 0$ ). Therefore, we extended our candidate model set to include the $\kappa-\mu$ extreme distribution. Using the $R A D$ to quantify the difference between the empirical and theoretical pdfs, it was found that modeling using the $\kappa-\mu$ extreme distribution represented the lowest information loss in $69 \%$ of the cases. Nevertheless, due to the close relationship between both models coupled with the severe fading experienced in this environment, the differences between both fits were almost visually indistinguishable in most cases.

Before concluding, it is worth remarking that the channel characteristics experienced for $\mathrm{W} 2 \mathrm{~W}$ systems operating in the presence of vehicular traffic can be expected to be different for dissimilar environments (e.g., city center, and urban and rural areas). Indeed, some work performed by the authors and not presented here for brevity has indicated that simple surrounding structures, such as a wall, can have a discernible effect on the link characteristics. In this paper, the presence of the wall promoted the impact of the vehicle, such that its effects were noticeable from a greater distance compared with the case when there are no surrounding structures. It also mitigated the shadowing effect of the vehicle as it transitioned the LOS path between the $\mathrm{W} 2 \mathrm{~W}$ users. In this paper, while we have focused solely on the impact of the vehicle on the $\mathrm{W} 2 \mathrm{~W}$ channel, as a future advancement of the work, we recommend that the $\mathrm{W} 2 \mathrm{~W}$ channel should be investigated from the environmental perspective to further enhance our knowledge of this emerging wireless application.

\section{REFERENCES}

[1] S. L. Cotton and W. G. Scanlon, "Using smart people to form future mobile wireless networks," Microw. J., vol. 54, pp. 24-40, Dec. 2011.

[2] J. Elias, S. Paris, and A. Mehaoua, "Cross technology interference mitigation in body-to-body area networks," in Proc. 14th Int. Symp. Workshops World Wireless, Mobile Multimedia Netw. (WoWMoM), Madrid, Spain, Jun. 2013, pp. 1-9.

[3] A. Argyriou, A. C. Breva, and M. Aoun, "Optimizing data forwarding from body area networks in the presence of body shadowing with dual wireless technology nodes," IEEE Trans. Mobile Comput., vol. 14, no. 3, pp. 632-645, Mar. 2015.

[4] M. A. Hanson et al., "Body area sensor networks: Challenges and opportunities," Computer, vol. 42, no. 1, pp. 58-65, 2009.

[5] K. M. S. Thotahewa, J. M. Redouté, and M. R. Yuce, "A low-power wearable dual-band wireless body area network system: Development and experimental evaluation," IEEE Trans. Microw. Theory Techn., vol. 62, no. 11, pp. 2802-2811, Nov. 2014.

[6] H.-R. Chuang, "Human operator coupling effects on radiation characteristics of a portable communication dipole antenna," IEEE Trans. Antennas Propag., vol. 42, no. 4, pp. 556-560, Apr. 1994.

[7] S. L. Cotton, "A statistical model for shadowed body-centric communications channels: Theory and validation," IEEE Trans. Antennas Propag., vol. 62, no. 2, pp. 1416-1424, Mar. 2014.

[8] P. Ferrand, J.-M. Gorce, and C. Goursaud, "On the packet error rate of correlated shadowing links in body-area networks," in Proc. 5th Eur. Conf. Antennas Propag. (EUCAP), Rome, Italy, Apr. 2011, pp. 3094-3098.

[9] G. Koutitas, "Multiple human effects in body area networks," IEEE Antennas Wireless Propag. Lett., vol. 9, pp. 938-941, Oct. 2010. 
[10] A. Fort, C. Desset, J. Ryckaert, P. De Doncker, L. Van Biesen, and P. Wambacq, "Characterization of the ultra wideband body area propagation channel," in Proc. IEEE Int. Conf. Ultra-Wideband, Zurich, Switzerland, Sep. 2005, p. 6.

[11] C. W. Kim, T. S. P. See, T. M. Chiam, Y. Ge, Z. N. Chen, and S. Sun, "Channel characterization of walking passerby's effects on $2.48-\mathrm{GHz}$ wireless body area network," IEEE Trans. Antennas Propag., vol. 61, no. 3, pp. 1495-1498, Mar. 2013.

[12] M. Jacob, C. Mbianke, and T. Kürner, "A dynamic $60 \mathrm{GHz}$ radio channel model for system level simulations with MAC protocols for IEEE 802.11ad," in Proc. IEEE 14th Int. Symp. Consum. Electron. (ISCE), Braunschweig, Germany, Jun. 2010, pp. 1-5.

[13] J. A. Rustako, M. J. Gans, G. J. Owens, and R. S. Roman, "Attenuation and diffraction effects from truck blockage of an 11-GHz line-of-sight microcellular mobile radio path," IEEE Trans. Veh. Technol., vol. 40, no. 1, pp. 211-215, Feb. 1991.

[14] R. Meireles, M. Boban, P. Steenkiste, O. Tonguz, and J. Barros, "Experimental study on the impact of vehicular obstructions in VANETs," in Proc. IEEE Veh. Netw. Conf. (VNC), Jersey City, NJ, USA, Dec. 2010, pp. 338-345.

[15] M. Boban, T. T. V. Vinhoza, M. Ferreira, J. Barros, and O. K. Tonguz, "Impact of vehicles as obstacles in vehicular ad hoc networks," IEEE J. Sel. Areas Commun., vol. 29, no. 1, pp. 15-28, Jan. 2011.

[16] R. He, A. F. Molisch, F. Tufvesson, Z. Zhong, B. Ai, and T. Zhang, "Vehicle-to-vehicle propagation models with large vehicle obstructions," IEEE Trans. Intell. Transp. Syst., vol. 15, no. 5, pp. 2237-2248, Oct. 2014.

[17] T. Abbas, F. Tufvesson, and J. Karedal. (Mar. 2012). "A measurement based shadow fading model for vehicle-to-vehicle network simulations." [Online]. Available: https://arxiv.org/abs/1203.3370

[18] H. Fernández, L. Rubio, V. M. Rodrigo-Peñarrocha, and J. Reig, "Path loss characterization for vehicular communications at $700 \mathrm{MHz}$ and $5.9 \mathrm{GHz}$ under LOS and NLOS conditions," IEEE Antennas Wireless Propag. Lett., vol. 13, pp. 931-934, May 2014.

[19] P. Paschalidis, K. Mahler, A. Kortke, M. Peter, and W. Keusgen, "Pathloss and multipath power decay of the wideband car-to-car channel at $5.7 \mathrm{GHz}$," in Proc. IEEE 73rd Veh. Technol. Conf. (VTC Spring), Budapest, Hungary, May 2011, pp. 1-5.

[20] M. G. Doone and S. L. Cotton, "Fading characteristics of body-to-body channels subject to vehicular traffic conditions at $2.45 \mathrm{GHz}, "$ in Proc. IEEE Antennas Propag. Soc. Int. Symp. (APSURSI), Memphis, TN, USA, Jul. 2014, pp. 723-724.

[21] M. G. Doone and S. L. Cotton, "Vehicular traffic intersecting bodyto-body communications channels at $2.45 \mathrm{GHz}$," in Proc. 9th Eur. Conf. Antennas Propag. (EUCAP), Lisbon, Portugal, Apr. 2015, pp. 1-4.

[22] Road Vehicles-Types-Terms and Definitions, document ISO 3833:1977, International Organization for Standardization, Geneva, Switzerland, 1997.

[23] Classes of Comparable Automobiles, document 4 C.F.R. §600.315-08, 2014.

[24] N. A. Dieng, M. Charbit, C. Chaudet, L. Toutain, and T. Ben Meriem, "Indoor localization in wireless networks based on a two-modes Gaussian mixture model," in Proc. IEEE Veh. Technol. Conf. (VTC Fall), Las Vegas, NV, USA, Sep. 2013, pp. 1-5.

[25] M.-M. Gao, C. Tai-Hua, and X.-X. Gao, "Application of Gaussian mixture model genetic algorithm in data stream clustering analysis," in Proc. IEEE Int. Conf. Intell. Comput. Intell. Syst. (ICIS), Xiamen, China, Oct. 2010, pp. 786-790.

[26] V. Cellini and G. Doná, "A novel joint channel and multi-user interference statistics estimator for UWB-IR based on Gaussian mixture model," in Proc. IEEE Int. Conf. Ultra-Wideband, Zurich, Switzerland, Sep. 2005, pp. 655-660.

[27] W. L. Martinez and A. R. Martinez, Computational Statistics Handbook With MATLAB. Boca Raton, FL, USA: CRC Press, 2001.

[28] P. Paalanen, "Bayesian classification using Gaussian mixture model and EM estimation: Implementations and comparisons," Dept. Inf. Technol., Lappeenranta Univ. Technol., Tech. Rep. 1, Jun. 2004.

[29] H. Bozdogan, "Model selection and Akaike's information criterion (AIC): The general theory and its analytical extensions," Psychometrika, vol. 52, no. 3, pp. 345-370, 1987.

[30] A. Fort, "Body area communications: Channel characterization and ultrawideband system-level approach for low power," Ph.D. dissertation, Vrije Univ. Brussel, Ixelles, Belgium, 2007.
[31] A. Fort, C. Desset, P. De Doncker, P. Wambacq, and L. Van Biesen, "An ultra-wideband body area propagation channel model-from statistics to implementation," IEEE Trans. Microw. Theory Techn., vol. 54, no. 4, pp. 1820-1826, Jun. 2006.

[32] M. D. Yacoub, "The $\kappa-\mu$ distribution and the $\eta-\mu$ distribution," IEEE Antennas Propag. Mag., vol. 49, no. 1, pp. 68-81, Feb. 2007.

[33] S. L. Cotton, "Human body shadowing in cellular device-to-device communications: Channel modeling using the shadowed $\kappa-\mu$ fading model," IEEE J. Sel. Areas Commun., vol. 33, no. 1, pp. 111-119, Jan. 2015

[34] D. W. Matolak and J. Frolik, "Worse-than-Rayleigh fading: Experimental results and theoretical models," IEEE Commun. Mag., vol. 49, no. 4, pp. 140-146, Apr. 2011.

[35] G. S. Rabelo, U. S. Dias, and M. D. Yacoub, "The $\kappa-\mu$ extreme distribution: Characterizing severe fading conditions," in IEEE MTT-S Int. Microw. Symp. Dig., Belem, Brazil, Nov. 2009, pp. 244-248.

[36] G. S. Rabelo and M. D. Yacoub, "The $\kappa-\mu$ extreme distribution," IEEE Trans. Commun., vol. 59, no. 10, pp. 2776-2785, Oct. 2011.

[37] D. Johnson and S. Sinanovic, "Symmetrizing the Kullback-Leibler distance," Rice Univ., Houston, TX, USA, Tech. Rep., 2001.

[38] B. Jiang, J. Pei, Y. Tao, and X. Lin, "Clustering uncertain data based on probability distribution similarity," IEEE Trans. Knowl. Data Eng., vol. 25, no. 4, pp. 751-763, Apr. 2013.

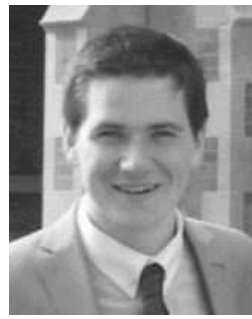

Michael G. Doone (S'15) received the M.Eng. degree (Hons.) in electrical and electronic engineering from Queen's University Belfast, Belfast, U.K., in 2013, where he is currently pursuing the $\mathrm{Ph} . \mathrm{D}$. degree in wireless vehicular communications channels involving people.

His current research interests include the characterization and modeling of wireless vehicular fading channels involving people, for both terrain-based and unmanned aerial vehicles, through bespoke test-bed systems and channel measurements.

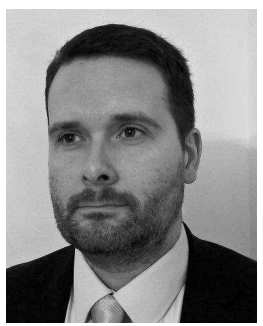

Simon L. Cotton (S'04-M'07-SM'14) received the B.Eng. degree in electronics and software from Ulster University, Ulster, U.K., in 2004, and the $\mathrm{Ph} . \mathrm{D}$. degree in electrical and electronic engineering from Queen's University Belfast, Belfast, U.K., in 2007.

$\mathrm{He}$ is currently a Reader of wireless communications with the Institute of Electronics, Communications and Information Technology, Queen's University Belfast, and also a Co-Founder and the Chief Technology Officer with ActivWireless Ltd., Belfast. He has authored or co-authored over 100 publications in major IEEE/IET journals and refereed international conferences, two book chapters, and two patents. His current research interests include cellular device-todevice, vehicular, and body-centric communications, radio channel characterization and modeling, and the simulation of wireless channels.

Dr. Cotton was a recipient of the H. A. Wheeler Prize, in 2010, from the IEEE Antennas and Propagation Society for the best applications journal paper in the IEEE TRANSACtions on ANTENNAS AND PROPAGation in 2009. In 2011, he was a recipient of the Sir George Macfarlane Award from the U.K. Royal Academy of Engineering in recognition of his technical and scientific attainment since graduating from his first degree in engineering.

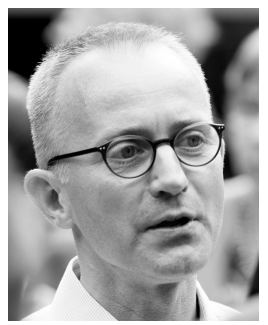

Claude Oestges (M'05-SM'12) received the M.Sc. and Ph.D. degrees in electrical engineering from the Université catholique de Louvain (UCL), Louvainla-Neuve, Belgium, in 1996 and 2000, respectively.

In 2001, he joined the Smart Antennas Research Group, Information Systems Laboratory, Stanford University, Stanford, CA, USA, as a Post-Doctoral Scholar. From 2002 to 2005, he was with the Microwave Laboratory, UCL, as a Post-Doctoral Fellow of the Belgian Fonds de la Recherche Scientifique. He is currently a Professor with the Electrical Engineering Department, Institute for Information and Communication Technologies, Electronics and Applied Mathematics, UCL. He has authored or co-authored three books and over 200 journal papers and conference communications.

Dr. Oestges was a recipient of the 1999-2000 IET Marconi Premium Award and the IEEE Vehicular Technology Society Neal Shepherd Award in 2004 and 2012, respectively. He currently serves as an Associate Editor of the IEEE TRANSACTIONS ON ANTENNAS AND PROPAGATION and the IEEE TRANSACTIONS ON VEHICULAR TECHNOLOGY. 